Gastroenterologe 2018 13:333-335 https://doi.org/10.1007/s11377-018-0281-6 Online publiziert: 26. Juli 2018

(c) Springer Medizin Verlag GmbH, ein Teil von Springer Nature 2018

CrossMark

\section{Ellenrieder ${ }^{1} \cdot$ P. Michl ${ }^{2}$}

${ }^{1}$ Klinik für Gastroenterologie und gastrointestinale Onkologie, Universitätsmedizin Göttingen (UMG), Göttingen, Deutschland

${ }^{2}$ Klinik für Innere Medizin I, Martin-Luther-Universität Halle/Wittenberg, Halle (Saale), Deutschland

\title{
Seltene maligne Erkrankungen des Gastrointestinaltrakts
}

Mehr als 100.000 Menschen erkranken in Deutschland jedes Jahr an einer seltenen Krebserkrankung. Damit sind seltene Krebsarten insgesamt zwar für mehr als $20 \%$ aller Krebserkrankungen verantwortlich, dennoch ist die Anzahl an Patienten, die an einer bestimmten seltenen Krebserkrankung leiden, nur sehr gering. Inzwischen sind mehr als 200 verschiedene seltene Krebsarten bekannt und viele hiervon sind im oberen, mittleren oder unteren Verdauungstrakt lokalisiert. Im klinischen Alltag stellen uns seltene gastrointestinale Tumorerkrankungen häufig vor größere diagnostische und therapeutische Herausforderungen und nicht selten erfolgt die bestmögliche Behandlung erst verzögert, weil die richtige Diagnose zu spät gestellt wird.

Die aktuelle Ausgabe der Zeitschrift Der Gastroenterologe hat sich diesem wichtigen Themenkomplex angenommen und in 5 Beiträgen renommierter Experten zentrale Aspekte der Pathogenese, klinischen Präsentation und der medikamentösen Behandlung einzelner seltener gastrointestinaler Tumorerkrankungen illustriert. Hierzu zählen beispielsweise gastrointestinale Stromatumoren (GIST), die aus Binde- oder Stützgewebe entstehen und für weniger als $1 \%$ der Tumoren des Magen-DarmTrakts verantwortlich sind. GIST-Tumoren gelten als Modellerkrankungen für zielgerichtete medikamentöse Therapien, die sich streng an der molekularen Diagnostik orientieren. Silke Cameron fasst in ihrem Beitrag die Bedeutung relevanter Tyrosinkinaserezeptormutationen für die Prognose und Therapieentscheidung zusammen und geht hierbei auch auf neue medikamentöse Strategien, wie z. B. epigenetische und immunonkologische Behandlungsprinzipien, ein. Gemischt neuroendokrin-nichtneuroendokrine Neoplasien (MiNEN) definieren eine neue Gruppe gastrointestinaler Tumorerkrankungen, die in der Regel aus schlecht differenzierten neuroendokrinen Anteilen und Komponenten von Adeno- oder Plattenepithelkarzinomen bestehen. "High-grade“ MiNEN kommen v.a. im Kolorektum vor und sind wahrscheinlich für mehr als ein Drittel aller neuroendokrinen Karzinome verantwortlich. Sebastian Krug und Patrick Michl erläutern in ihrem Artikel nicht nur die aktuellen Klassifikationsund Diagnosekriterien sowie klinische Charakteristika von MiNEN, sondern stellen auch in sehr anschaulicher Weise die Evidenzlage gegenwärtiger therapeutischer Möglichkeiten in der Behandlung dieser seltenen Tumorerkrankungen dar. Neben dem duktalen Pankreasadenokarzinom zählt das Azinuszellkarzinom zu den soliden malignen Erkrankungen der Bauchspeicheldrüse. Dabei ist das Azinuszellkarzinom deutlich seltener und nur für etwa $1 \%$ aller exokrinen Pankreaskarzinome verantwortlich. Christian Bauer und Thomas Gress erarbeiten in ihrem Beitrag den aktuellen Stand der molekularen Karzinogenese und fassen die genetischen, klinischen und therapeutischen Besonderheiten des Azinuszellkarzinoms zusammen. Interessant ist hierbei das gehäufte Auftreten genetischer Defekte im DNA-mismatchrepair(MMR)-System, das eine wirksame Therapie mit Checkpointinhibitoren ermöglicht. Das Cronkhite-Canada-Syndrom zählt zu den seltenen Formen der intestinalen Polyposis. Weltweit sind bislang nur etwa 500 Fälle beschrieben worden. In ihrem Artikel schildern Nina Gliem und Volker Ellenrieder einen interessanten Fall eines Patienten mit Cronkhite-Canada-Syndrom, der durch eine progrediente klinische Symptomatik mit Geschmackstörungen, ausgeprägten Onychodystrophien und Hyperpigmentationen auffällig wurde. Die koloskopische Abklärung erbrachte den Nachweis multipler Polypen, die histologisch Hamartomen entsprachen. Gliem und Ellenrieder grenzen in ihrem Bericht nicht nur das erworbene Cronkhite-Canada-Syndrom von genetisch definierten Polyposissyndromen $a b$, sondern gehen auch auf den aktuellen Stand möglicher Behandlungsoptionen ein. Das intrahepatische Gallengangskarzinom gehört trotz steigender Inzidenz ebenfalls zur Gruppe der seltenen Krebserkrankungen. Die Prognose ist mit einem medianen Überleben von etwa 11 Monaten infaust und die Erfolge einer konventionellen zytostatischen Therapie beim fortgeschrittenen Karzinom sind schlecht. Andrea Sabarowski und Arndt Vogel fassen den aktuellen Wissensstand zu Diagnostik und Therapie zusammen und weisen auf ermutigende neue Untersuchungsergebnisse bei Patienten mit spezifischen genetischen Veränderungen (IDH1-Mutation bzw. FGFR2-Fusion) hin.

Mit dieser Schwerpunktausgabe verfolgen die Herausgeber von Der Gastroenterologe das Ziel, auf seltene Tumorerkrankungen des Gastrointestinaltrakts hinzuweisen, die häufig übersehen oder zu spät diagnostiziert werden. Wir hoffen sehr, dass die Wahl der Beiträge und 
ihre Darstellung das Interesse der Leser finden.

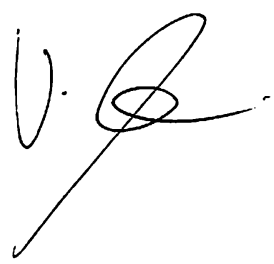

Prof. Dr. Volker Ellenrieder

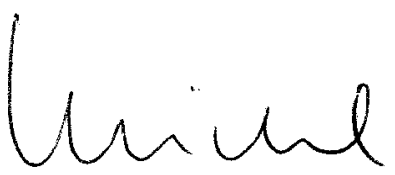

Prof. Dr. Patrick Michl

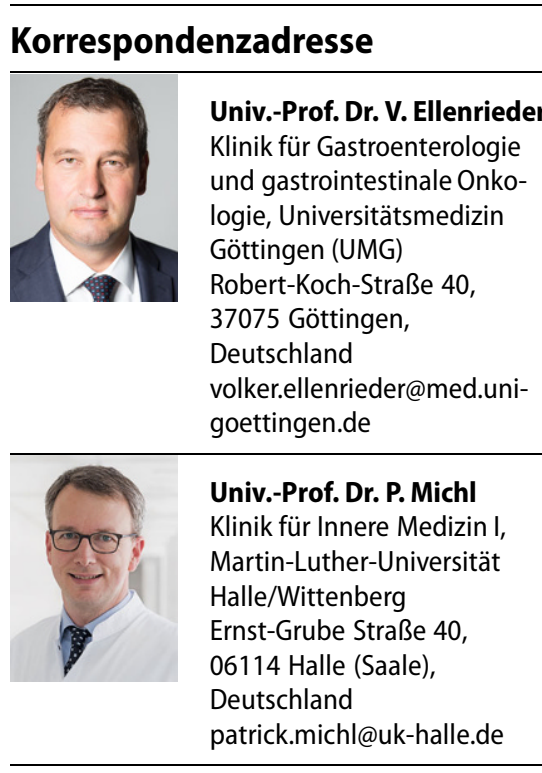

Interessenkonflikt. V. Ellenrieder und P. Michl geben an, dass kein Interessenkonflikt besteht.

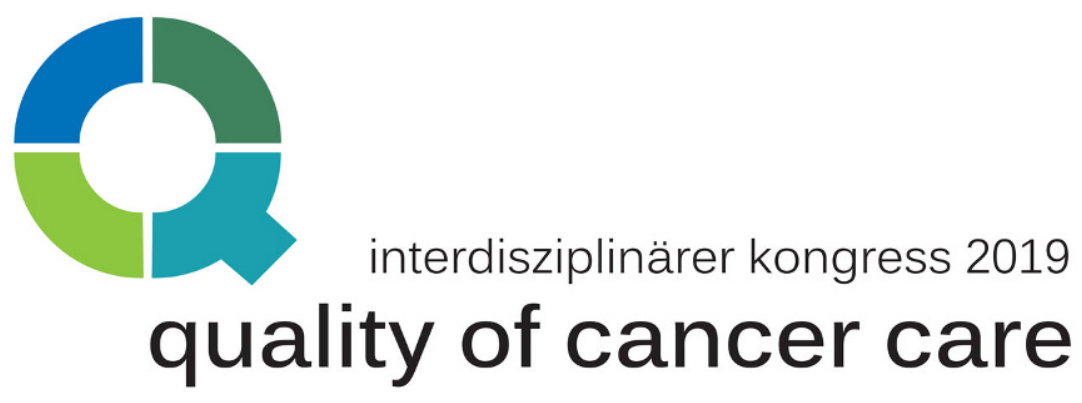

\section{Qualität heißt: aus der Versorgung lernen}

\section{Abstracteinreichung für den 4. interdisziplinären Kongress Quality of Cancer Care} ist gestartet

In Vorbereitung des 4. interdisziplinären Kongresses Quality of Cancer Care

(QoCC) im März 2019 in Berlin hat die wissenschaftliche Leitung zur Abstracteinreichung aufgerufen. Die Einreichung erfolgt in der Zeit bis 15 . November 2018 über die Kongresswebseite www.qocc.de/abstracts.html. Elf Kategorien sind formuliert - von Qualitätsforschung bis Leitlinienimplementierung. Ausdrücklich sind Nachwuchswissenschaftlerinnen und -wissenschaftler zur Einreichung aufgerufen. Drei Abstracts werden das Kongressprogramm am 28. und 29. März 2019 als Best-of-Abstracts-Vortrag erweitern. Die anderen angenommenen Abstracts werden an beiden Kongresstagen in Rahmen von geführten Posterbegehungen präsentiert.

Der QoCC ist das Forum für onkologische Versorgungsforschung und systematische Qualitätsentwicklung in der Onkologie in Deutschland. Er ist eine hervorragende Gelegenheit, eigene Daten vorzustellen und andere Forscher zu fachlichen Diskussionen und weiteren Untersuchungen anzuregen. Darauf verweist auch das 2019er Motto "Qualität heißt: aus der Versorgung lernen“. QoCCTeilnehmer sind Experten von Krebsregistern, Tumorzentren, zertifizierten Zentren, Clinical-Research-Organisationen, Einrichtungen der Versorgungsforschung, Epidemiologen, Biometriker, Gesundheitsökonomen, Kliniker und Gesundheitspolitiker.

Ausrichter des QoCC sind die Deutsche Krebsgesellschaft e. V. und die Arbeitsgemeinschaft Deutscher Tumorzentren e. V. Anders als andere Veranstaltungen greift der QoCC keine einzelnen Aspekte auf, vielmehr geht es um eine Verknüpfung von onkologischen
Leitlinien, Zertifizierung und Dokumentation, klinischen Studien und Versorgungsforschung. Die Idee zu diesem Kongress entstand aus der Erfahrung, dass es häufig die Schnittstellen zwischen den Bereichen sind, an denen es in der Qualitätsentwicklung der Krebsversorgung zu Reibungsverlusten kommt. Das gewählte Format des QoCC, eine Kombination aus Tutorials, Vorträgen, Diskussionsrunden und Posterpräsentationen, bietet eine ausgezeichnete Plattform für einen konstruktiven Gedanken- und Erfahrungsaustausch zur Minimierung von Hindernissen und Reibungsverlusten.

Renate Babnik, Referentin Kommunikation in der Deutschen Krebsgesellschaft e. V., babnik@krebsgesellschaft.de

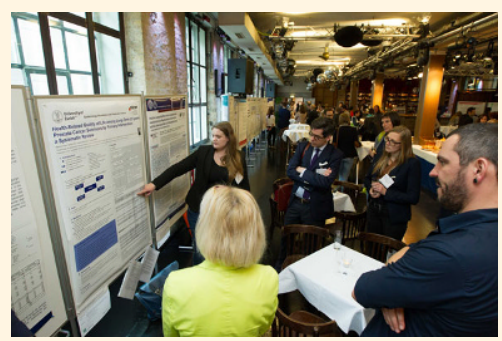

Posterbegehung beim QoCC 2017. (Quelle: berlinevent-foto.de) 
Hier steht eine Anzeige.

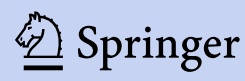

